\title{
THE AMPLIFIED QUANTUM FOURIER TRANSFORM: SOLVING THE LOCAL PERIOD PROBLEM
}

\author{
DAVID J. CORNWELL
}

\begin{abstract}
This paper creates and analyses a new quantum algorithm called the Amplified Quantum Fourier Transform (Amplified-QFT) for solving the following problem:

The Local Period Problem: Let $L=\{0,1 \ldots N-1\}$ be a set of $N$ labels and let $A$ be a subset of $M$ labels of period $P$, i.e. a subset of the form $A=\{j: j=s+r P, r=0,1 \ldots M-1\}$ where $P \leq \sqrt{N}$ and $M<<N$, and where $M$ is assumed known. Given an oracle $f: L \rightarrow\{0,1\}$ which is 1 on $A$ and 0 elsewhere, find the local period $P$. A separate algorithm finds the offset $s$.

The first part of this paper defines the Amplified-QFT algorithm. The second part of the paper summarizes the main results and compares the Amplified-QFT algorithm against the Quantum Fourier Transform (QFT) and Quantum Hidden Subgroup (QHS) algorithms when solving the local period problem. It is shown that the Amplified-QFT algorithm is, on average, quadratically faster than both the QFT and QHS algorithms. The third part of the paper provides the detailed proofs of the main results, describes the method of recovering $P$ from an observation $y$ and describes the algorithm for finding the offset $s$.
\end{abstract}

Acknowledgement 1. I would like to thank my advisor, Professor Samuel J. Lomonaco of the CSEE Department of the University of Maryland Baltimore County $(U M B C)$ for his guidance and help in the writing of this paper.

\section{Introduction}

This paper creates and analyzes a new quantum algorithm called the Amplified Quantum Fourier Transform (Amplified-QFT) for solving the following problem:

The Local Period Problem: Let $L=\{0,1 \ldots N-1\}$ be a set of $N$ labels and let $A$ be a subset of $M$ labels of period $P$, i.e. a subset of the form $A=\{j: j=$ $s+r P, r=0,1 \ldots M-1\}$ where $P \leq \sqrt{N}$ and $M<<N$, and where $M$ is assumed known. Given an oracle $f: L \rightarrow\{0,1\}$ which is 1 on $A$ and 0 elsewhere, find the local period $P$. A separate algorithm finds the offset $s$.

The first part of this paper defines the Amplified-QFT algorithm. The second part of the paper summarizes the main results and compares the Amplified-QFT algorithm against the Quantum Fourier Transform (QFT) and Quantum Hidden Subgroup (QHS) algorithms when solving the local period problem. It is shown that the Amplified-QFT algorithm is, on average, quadratically faster than both the QFT and QHS algorithms. The third part of the paper provides the detailed

Date: September 30th, 2010, Accepted into QIP Journal August 2nd, 2012.

2000 Mathematics Subject Classification. Primary 05C38, 15A15; Secondary 05A15, 15A18.

Key words and phrases. Quantum Fourier Transform, Amplitude Amplification, Oracle, Period Finding, Shor Algorithm, Grover Algorithm. 
proofs of the main results, describes the method of recovering $P$ from an observation $y$ and describes the algorithm for finding the offset $s$.

\section{Background-Amplitude Amplification}

In ref[4] Lov Grover specified a quantum search algorithm that searched for a single marked element $x 0$ in an $N$ long list $L$. An oracle $f: L \rightarrow\{0,1\}$ is used to mark the element such that $f(x 0)=1$ and $f$ is 0 elsewhere. Grover's quantum algorithm finds the element with a work factor of $O(\sqrt{N})$ whereas on a classical computer this would take $O(N)$, thereby obtaining a quadratic speedup. Grover's algorithm can be summarized as follows:

a) Initialize the state to be the uniform superposition state $|\psi>=H| 0>$ where $H$ is the Hadamard transform.

b) Reflect the current state about the plane orthogonal to the state $\mid x 0>$ by using the operator $(I-2|x 0><x 0|)$.

c) Reflect the new state back around $\mid \psi>$ by using the operator $(2|\psi><\psi|-I)$. This operator is a reflection about the average of the amplitudes of the new state.

d) Repeat steps b) and c) $O(\sqrt{N})$ times until most of the probability is on $\mid x 0>$.

e) Measure the resulting state to obtain $x 0$.

Also in ref[4], Grover suggested this algorithm could be extended to the case of searching for an element in a subset $A$ of $M$ marked elements in an $N$ long list $L$. Once again an oracle $f: L \rightarrow\{0,1\}$ is used to mark the elements of the subset $A$.Grover's algorithm solves this problem with a work factor of $O(\sqrt{N / M})$. The elements of the set $A$ are sometimes referred to as "good" and the elements not in $A$ are called "bad". Grover's algorithm for this problem can be summarized as follows:

a) Initialize the state to be the uniform superposition state $|\psi\rangle=H \mid 0>$ where $H$ is the Hadamard transform.

b) Reflect the current state about the plane orthogonal to the state $\mid x \operatorname{good}>$ by using the operator $(I-2 \mid x$ good $><x$ good $\mid)$, where $x$ good is the normalized sum of the good states defined by the set $A$.This changes the sign of the amplitudes of the good states defined by $A$.

c) Reflect the new state back around $\mid \psi>$ by using the operator $(2|\psi><\psi|-I)$.

d) Repeat steps b) and c) $O(\sqrt{N / M})$ times until most of the probability is on the set $A$.

e) Measure the resulting state to obtain an element in the set $A$.

Both versions of Grover's algorithm are also known as Amplitude Amplification algorithms. which are generalized even further in ref [9]. The first part of the Amplified-QFT algorithm consists of the second of these algorithms, except for the final measurement step e).

\section{BACKGROUND-PERIOd Finding}

In ref[3], Peter Shor describes a quantum algorithm to solve the factorization problem with exponential speed up over classical approaches. He translates the factorization problem into one of finding the period of the function $a^{x} \operatorname{Mod} N$ where $N$ is the number to be factored and $\operatorname{gcd}(a, N)=1$. The period is found by making use of the QFT. Shor's factorization algorithm is summarized below:

a) Find $Q: N^{2} \leq Q<2 N^{2}$

b) Find $a: \operatorname{gcd}(\bar{a}, N)=1$ 
c) Find the period of $a^{x} M o d N$ using the QFT and using the $Q t \dot{h}$ root of unity

- Form the superposition $\frac{1}{\sqrt{Q}} \sum|x>| a^{x} \operatorname{ModN}>$

- Apply the QFT to the first register $\left|x>\rightarrow \sum \omega^{x y}\right| y>$

- Measure $y$

- Form the continued fraction expansion of $y / Q$ to find $d / P$

- If $|y / Q-d / P|<1 / 2 N^{2}$ and $\operatorname{gcd}(d, P)=1$ then $P$ is recovered

d) If the period is not even start over

e) If $a^{P / 2}+1=0 \operatorname{Mod} N$ start over

f) Find $\operatorname{gcd}\left(a^{p / 2}-1, N\right)$ to find the factor of $N$.

Step c) is the quantum part of Shor's factorization algorithm. We make use of the QFT and continued fraction expansion method to recover the period $P$ in the second part of the Amplified-QFT algorithm.

\section{The Amplified Quantum Fourier Transform Algorithm}

The Amplified-QFT algorithm solves the Local Period Problem:

The Local Period Problem: Let $L=\{0,1 \ldots N-1\}$ be a set of $N$ labels and let $A$ be a subset of $M$ labels of period $P$, i.e. a subset of the form $A=\{j: j=$ $s+r P, r=0,1 \ldots M-1\}$ where $P \leq \sqrt{N}$ and $M<<N$, and where $M$ is assumed known. Given an oracle $f: L \rightarrow\{0,1\}$ which is 1 on $A$ and 0 elsewhere, find the local period $P$. A separate algorithm finds the offset $s$.

The Amplified-QFT algorithm consists of the following steps where steps a) through d) are the Amplitude Amplification steps and steps e) through h) are the period finding steps that use the QFT:

a) Initialize the state to be the uniform superposition state $|\psi>=H| 0>$ where $H$ is the Hadamard transform.

b) Reflect the current state about the plane orthogonal to the state $\mid x$ good $>$ by using the operator $(I-2 \mid x$ good $><x$ good $\mid)$, where $x$ good is the normalized sum of the good states defined by the set $A$.This changes the sign of the amplitudes of the good states defined by $A$.

c) Reflect the new state back around $\mid \psi>$ by using the operator $(2|\psi><\psi|-I)$.

d) Repeat steps b) and c) $O(\sqrt{N / M})$ times until most of the probability is on the set $A$.

e) Apply the QFT to the resulting state

f) Make a measurement $y$

g) Form the continued fraction expansion of $y / N$ to find $d / P$

h) If $|y / N-d / P|<1 / 2 N^{2}$ and $\operatorname{gcd}(d, P)=1$ then $P$ is recovered

i) If $\operatorname{gcd}(d, P) \neq 1$ repeat the algorithm starting at step a)

The Amplified-QFT algorithm produces the following states (See later sections for the detailed analysis of the Amplified-QFT algorithm):

After applying steps b) and c) $k$ times where $k=\left\lfloor\frac{\pi}{4 \sin ^{-1}(\sqrt{M / N})}\right\rfloor$ we arrive at the following state:

$$
\left|\psi_{k}>=a_{k} \sum_{z \in A}\right| z>+b_{k} \sum_{z \notin A} \mid z>
$$

where

$$
a_{k}=\frac{1}{\sqrt{M}} \sin (2 k+1) \theta, b_{k}=\frac{1}{\sqrt{N-M}} \cos (2 k+1) \theta
$$


are the appropriate amplitudes of the states and where

$$
\sin \theta=\sqrt{M / N}, \cos \theta=\sqrt{1-M / N}
$$

The QFT at step e) performs the following action

$$
\left|z>\rightarrow \frac{1}{\sqrt{N}} \sum_{y=0}^{N-1} e^{-2 \pi i z y / N}\right| y>
$$

After the application of the QFT to the state $\mid \psi_{k}>$, letting $\omega=e^{-2 \pi i / N}$ we arrive at the following sate:

$$
\left|\phi_{k}>=\sum_{y=0}^{N-1}\left[\frac{a_{k}}{\sqrt{N}} \sum_{z \in A} \omega^{z y}+\frac{b_{k}}{\sqrt{N}} \sum_{z \notin A} \omega^{z y}\right]\right| y>
$$

At step f) we measure this state with respect to the standard basis to yield an integer $y \in\{0,1, \ldots, N-1\}$ from which we can determine the period $P$ using the continued fraction method.

In a later section where we summarize the main results, we provide a table showing the probabilities of measuring $y$ for the Amplified-QFT algorithm and compare them against the probabilites obtained by performing the QFT and QHS algorithms.

\section{The QFT Algorithm}

The QFT algorithm does not include the amplitude amplification steps and consists of the following steps:

a) Initialize the state to be the uniform superposition state $|\psi\rangle=H \mid 0>$ where $H$ is the Hadamard transform.

b) Apply the oracle $f$ to $|\psi\rangle$

c) Apply the QFT to this state

d) Make a measurement $y$

e) Form the continued fraction expansion of $y / N$ to find $d / P$

f) If $|y / N-d / P|<1 / 2 N^{2}$ and $\operatorname{gcd}(d, P)=1$ then $P$ is recovered

g) If $\operatorname{gcd}(d, P) \neq 1$ repeat the algorithm starting at step a)

At step b) after applying the oracle the state is given by (See later sections for the detailed analysis of the QFT algorithm):

$$
\mid \psi_{1}>=\frac{1}{\sqrt{N}}\left[(-2) \sum_{z \in A}\left|z>+\sum_{z=0}^{N-1}\right| z>\right]
$$

At step c) the QFT applies the following action:

$$
\left|z>\rightarrow \frac{1}{\sqrt{N}} \sum_{y=0}^{N-1} \omega^{z y}\right| y>
$$

to get

$$
\left|\psi_{2}>=\sum_{y=0}^{N-1}\left[\frac{(-2)}{N} \sum_{z \in A} \omega^{z y}+\frac{1}{N} \sum_{z=0}^{N-1} \omega^{z y}\right]\right| y>
$$


At step d) we measure this state with respect to the standard basis to yield an integer $y \in\{0,1, \ldots, N-1\}$ from which we can determine the period $P$ using the continued fraction method.

\section{The QHS Algorithm}

The QHS algorithm is a two register algorithm and does not include the amplitude amplification steps. It consists of the following steps:

a) Initialize the state to be the uniform superposition state $|\psi>=H| 0>\mid 0>$ where $H$ is the Hadamard transform.

b) Apply the oracle $f$ and put the result into the second register of $\mid \psi>$

c) Apply the QFT to the first register of this state

d) Make a measurement $y$

e) Form the continued fraction expansion of $y / N$ to find $d / P$

f) If $|y / N-d / P|<1 / 2 N^{2}$ and $\operatorname{gcd}(d, P)=1$ then $P$ is recovered

g) If $\operatorname{gcd}(d, P) \neq 1$ repeat the algorithm starting at step a)

At step b) we have the following state (See later sections for the detailed analysis of the QHS algorithm):

$$
\left|\psi_{1}>=\frac{1}{\sqrt{N}} \sum_{x=0}^{N-1}\right| x>\mid f(x)>
$$

After applying the QFT the state is given by:

$$
\left|\psi_{2}>=\sum_{y=0}^{N-1} \frac{1}{N}\right| y>\sum_{x=0}^{N-1} \omega^{x y} \mid f(x)>
$$

At step d) we measure this state with respect to the standard basis to yield an integer $y \in\{0,1, \ldots, N-1\}$ from which we can determine the period $P$ using the continued fraction method.

\section{Summary of the Main Results}

We summarize the main results and compare the probability $\operatorname{Pr}(y)$ of measuring $y$ in the final state arrived at for each of the three algorithms: 1) the Amplified-QFT algorithm 2) the QFT algorithm and 3) the QHS algorithm. Here $\sin \theta=\sqrt{M / N}$ and $k=\left\lfloor\frac{\pi}{4 \theta}\right\rfloor$ and $0 \leq \frac{\sin ^{2}(\pi M P y / N)}{\sin ^{2}(\pi P y / N)} \leq M^{2}$.

Case 1 (Amplified-QFT):

The probability $\operatorname{Pr}(y)$ is given exactly by

$$
\left\{\begin{array}{ll}
\cos ^{2} 2 k \theta & \text { if } \quad y=0 \\
\tan ^{2} \theta \sin ^{2} 2 k \theta & \text { if } \quad P y=0 \bmod N, y \neq 0 \\
\frac{1}{M^{2}} \tan ^{2} \theta \sin ^{2} 2 k \theta \frac{\sin ^{2}(\pi M P y / N)}{\sin ^{2}(\pi P y / N)} & \text { if } \quad P y \neq 0 \bmod N \text { and } M P y \neq 0 \bmod N \\
0 & \text { if } \quad P y \neq 0 \bmod N \text { and } M P y=0 \bmod N
\end{array}\right\}
$$


Case $2(\mathrm{QFT})$ :

The probability $\operatorname{Pr}(y)$ is given exactly by

$$
\left\{\begin{array}{ll}
\left(1-\frac{2 M}{N}\right)^{2} & \text { if } \quad y=0 \\
4 \frac{M^{2}}{N^{2}} & \text { if } \quad P y=0 \bmod N, y \neq 0 \\
\frac{4}{N^{2}} \frac{\sin ^{2}(\pi M P y / N)}{\sin ^{2}(\pi P y / N)} & \text { if } \quad P y \neq 0 \bmod N \text { and } M P y \neq 0 \bmod N \\
0 & \text { if } \quad P y \neq 0 \bmod N \text { and } M P y=0 \bmod N
\end{array}\right\}
$$

Case 3 (QHS):

The probability $\operatorname{Pr}(y)$ is given exactly by

$$
\left\{\begin{array}{ll}
1-\frac{2 M(N-M)}{N^{2}} & \text { if } \quad y=0 \\
\frac{2 M^{2}}{N^{2}} & \text { if } \quad P y=0 \bmod N, y \neq 0 \\
\frac{2}{N^{2}} \frac{\sin ^{2}(\pi M P y / N)}{\sin ^{2}(\pi P y / N)} & \text { if } \quad P y \neq 0 \bmod N \text { and } M P y \neq 0 \bmod N \\
0 & \text { if } \quad P y \neq 0 \bmod N \text { and } M P y=0 \bmod N
\end{array}\right\}
$$

We note that for the QFT and QHS algorithms $\operatorname{Pr}(y=0)$ is very close to 1 because $M<<N$. In the cases where $y \neq 0$ we compare the ratios of $\operatorname{Pr}(y)$ in the Amplified-QFT and QFT case and then in the Amplified-QFT and QHS case. Let $y$ be fixed such that either

1. $P y=0 \bmod N, y \neq 0$ or

2. $P y \neq 0 \bmod N$ and $M P y \neq 0 \bmod N$

and define $\operatorname{Pr} \operatorname{Ratio}(y)=\operatorname{Pr}(y)_{\text {Amplified-QFT }} / \operatorname{Pr}(y)_{Q F T}$ then we have the following (see the later detailed sections)

$$
\begin{aligned}
\frac{N}{4 M}\left(\frac{N}{N-M}\right) & \geq \operatorname{Pr} \operatorname{Ratio}(y) \geq \frac{N}{4 M}\left(\frac{N}{N-M}\right)\left(1-\frac{2 M}{N}\right)^{2} \\
& \Longrightarrow \operatorname{Pr} \operatorname{Ratio}(y) \approx \frac{N}{4 M}
\end{aligned}
$$

and define $\operatorname{Pr} \operatorname{Ratio}(y)=\operatorname{Pr}(y)_{\text {Amplified-QFT }} / \operatorname{Pr}(y)_{Q H S}$ then we have the following

$$
\begin{aligned}
\frac{N}{2 M}\left(\frac{N}{N-M}\right) & \geq \operatorname{Pr} \operatorname{Ratio}(y) \geq \frac{N}{2 M}\left(\frac{N}{N-M}\right)\left(1-\frac{2 M}{N}\right)^{2} \\
& \Longrightarrow \operatorname{Pr} \operatorname{Ratio}(y) \approx \frac{N}{2 M}
\end{aligned}
$$

Let $S_{A L G}=\left\{y:\left|\frac{y}{N}-\frac{d}{P}\right| \leq \frac{1}{2 P^{2}},(d, P)=1\right\}$ be the set of "successful" $y$ 's. That is $S_{A L G}$ consists of those $y$ 's which can be measured after applying one of the three algorithms denoted by $A L G$ and from which the period $P$ can be recovered by the method of continued fractions. Note that the set $S_{A L G}$ is the same for each 
algorithm. However the probability of this set varies with each algorithm. We can see from the following that given $y 1$ and $y 2$, whose probability ratios satisfy the same inequality, we can add their probabilities to get a new ratio that satisfies the same inequality. In this way we can add probabilities over a set on the numerator and denominator and maintain the inequality:

$$
\begin{aligned}
A & >\frac{P(y 1)}{Q(y 1)}>B \text { and } A>\frac{P(y 2)}{Q(y 2)}>B \\
& \Longrightarrow A>\frac{P(y 1)+P(y 2)}{Q(y 1)+Q(y 2)}>B
\end{aligned}
$$

We see from the cases given above that

$$
\frac{N}{4 M}\left(\frac{N}{N-M}\right) \geq \frac{\operatorname{Pr}\left(S_{\text {Amplified }-Q F T}\right)}{\operatorname{Pr}\left(S_{Q F T}\right)} \geq \frac{N}{4 M}\left(\frac{N}{N-M}\right)\left(1-\frac{2 M}{N}\right)^{2}
$$

where the difference between the upper bound and lower bound is exactly 1 and that

$$
\frac{N}{2 M}\left(\frac{N}{N-M}\right) \geq \frac{\operatorname{Pr}\left(S_{\text {Amplified-QFT }}\right)}{\operatorname{Pr}\left(S_{Q H S}\right)} \geq \frac{N}{2 M}\left(\frac{N}{N-M}\right)\left(1-\frac{2 M}{N}\right)^{2}
$$

where the difference between the upper bound and lower bound is exactly 2 .

This shows that the Amplified-QFT is approximately $\frac{N}{4 M}$ times more successful than the QFT and $\frac{N}{2 M}$ times more successful than the QHS when $M<<N$. In addition it also shows that the QFT is 2 times more successful than the QHS in this problem. However, the success of the Amplified-QFT algorithms comes at an increase in work factor of $O\left(\sqrt{\frac{N}{M}}\right)$. We note that in the case that $\mathrm{P}$ is a prime number that $(d, P)=1$ is met trivially. However when $\mathrm{P}$ is composite the algorithms may need to be rerun several times until $(d, P)=1$ is satisfied.

Towards the end of the paper we show how to test whether a putative value of $P$, given $s$ is known, can be tested to see if it is the correct value. We also investigate the case where $s$ is unknown but is from a small known set of values such that the values of $s$ can be exhausted over on a classical computer. We also show how $s$ can be recovered by using a quantum algorithm using amplitude amplification followed by a measurement.

\section{The Amplified-QFT is Quadratically Faster than the QFT or the QHS}

We show that the Amplified-QFT algorithm is, on average, quadratically faster than the QFT or QHS algorithms. In order to show this, we use the geometric probability distribution which provides the probability of the first success in a sequence of trials where the probability of success is $p$ and the probability of failure is $1-p$. For both the QFT and QHS algorithms a trial is one complete execution of the algorithm. Because the probability of measuring $y=0$ is close to 1 we expect to have to repeat the algorithm many times due to failure of measuring a successful $y$, before we have the first success.

If $X$ is the random variable which counts the number of trials until the first success then

$$
P(X=k)=(1-p)^{k-1} p \text { for } k=1,2 \ldots
$$


The expected value $E[X]$ and variance $\operatorname{Var}[X]$ are given by:

$$
E[X]=\frac{1}{p} \text { and } \operatorname{Var}[X]=\frac{1-p}{p^{2}}
$$

The workfactor of the Amplified-QFT algorithm is given by the number of iterations of each amplification step followed by a single QFT step:

$$
O\left(\sqrt{\frac{N}{M}}\right)
$$

For the QFT algorithm we have the probability of failure $1-p$ is given by

then

$$
\operatorname{Pr}(\text { failure })=1-p \geq \operatorname{Pr}(y=0)=\left(1-\frac{2 M}{N}\right)^{2}
$$

$$
\operatorname{Pr}(\text { success })=p \leq 1-\left(1-\frac{2 M}{N}\right)^{2}=\frac{4 M}{N}\left(1-\frac{M}{N}\right)
$$

Then for the QFT algorithm, the expected number of trials until the first success is

$$
E[X]=\frac{1}{p} \geq \frac{N}{4 M\left(1-\frac{M}{N}\right)} \geq \frac{N}{4 M}
$$

The workfactor of the QFT algorithm is the expected number of times the QFT has to be run, is given approximately by:

$$
O\left(\frac{N}{M}\right)
$$

Therefore the ratio of the expected work factor of the QFT algorithm and the work factor of the Amplified-QFT is given by

$$
O\left(\sqrt{\frac{N}{M}}\right)
$$

showing that the Amplified-QFT algorithm is, on average, quadratically faster than the QFT algorithm.

The variance in the number of times the QFT algorithm is run is given by

$$
\operatorname{Var}[X]=\frac{1-p}{p^{2}} \geq\left(\frac{N}{N-M}\right)^{2}\left(\frac{N-2 M}{4 M}\right)^{2}
$$

For the QHS algorithm we have the probability of failure $1-p$ is given by

then

$$
\operatorname{Pr}(\text { failure })=1-p \geq \operatorname{Pr}(y=0)=1-\frac{2 M(N-M)}{N^{2}}
$$

$$
\operatorname{Pr}(\text { success })=p \leq 1-\left(1-\frac{2 M(N-M)}{N^{2}}\right)=\frac{2 M}{N}\left(1-\frac{M}{N}\right)
$$

Then for the QHS algorithm, the expected number of trials until the first success is

$$
E[X]=\frac{1}{p} \geq \frac{N}{2 M\left(1-\frac{M}{N}\right)} \geq \frac{N}{2 M}
$$


The workfactor of the QHS algorithm is the expected number of times the QHS has to be run, is given approximately by:

$$
O\left(\frac{N}{M}\right)
$$

Therefore the ratio of the expected work factor of the QHS algorithm and the work factor of the Amplified-QFT is given by

$$
O\left(\sqrt{\frac{N}{M}}\right)
$$

showing that the Amplified-QFT algorithm is, on average, quadratically faster than the QHS algorithm.

The variance in the number of times the QHS algorithm is run is given by

$$
\operatorname{Var}[X]=\frac{1-p}{p^{2}} \geq\left(\frac{N}{N-M}\right)^{2}\left(\frac{(N-M)^{2}+M^{2}}{4 M^{2}}\right)
$$

\section{The Amplified-QFT Algorithm - Detailed Analysis}

In this section we examine the Amplified-QFT algorithm in detail and produce the results for the probability of success that were summarized earlier in the paper.

The Amplified-QFT algorithm is defined by the following procedure (see earlier section):

Steps a) to d): Apply the Amplitude Amplification algorithm to the starting state $\mid 0>$. The resulting state is given by $\mid \psi_{k}>(\operatorname{ref}[4], \operatorname{ref}[7], \operatorname{ref}[1])$ where $k=$ $\left\lfloor\frac{\pi}{4 \sin ^{-1}(\sqrt{M / N})}\right\rfloor:$

$$
\left|\psi_{k}>=a_{k} \sum_{z \in A}\right| z>+b_{k} \sum_{z \notin A} \mid z>
$$

where

$$
a_{k}=\frac{1}{\sqrt{M}} \sin (2 k+1) \theta, b_{k}=\frac{1}{\sqrt{N-M}} \cos (2 k+1) \theta
$$

are the appropriate amplitudes of the states and where

$$
\sin \theta=\sqrt{M / N}, \cos \theta=\sqrt{1-M / N}
$$

Now we have, $\operatorname{ref}[7]$,

$$
\begin{aligned}
& k=\left\lfloor\frac{\pi}{4 \theta}\right\rfloor \Longrightarrow \frac{\pi}{4 \theta}-1 \leq k \leq \frac{\pi}{4 \theta} \Longrightarrow \frac{\pi}{2}-\theta \leq(2 k+1) \theta \leq \frac{\pi}{2}+\theta \\
& \Longrightarrow \sin \theta=\cos \left(\frac{\pi}{2}-\theta\right) \geq \cos (2 k+1) \theta \geq \cos \left(\frac{\pi}{2}+\theta\right)=-\sin \theta
\end{aligned}
$$

Notice that the total probability of the N-M labels that are not in A is

$$
\begin{aligned}
(N-M)\left(\frac{1}{\sqrt{N-M}} \cos (2 k+1) \theta\right)^{2} & =\cos ^{2}(2 k+1) \theta \\
& \Longrightarrow \cos ^{2}(2 k+1) \theta \leq \sin ^{2} \theta=\sin ^{2}\left(\sin ^{-1}\left(\sqrt{\frac{M}{N}}\right)\right) \\
& \Longrightarrow \cos ^{2}(2 k+1) \theta \leq \frac{M}{N}
\end{aligned}
$$


whereas the total probability of the $\mathrm{M}$ labels in $\mathrm{A}$ is

$$
\begin{aligned}
M\left(\frac{1}{\sqrt{M}} \sin (2 k+1) \theta\right)^{2} & =\sin ^{2}(2 k+1) \theta=1-\cos ^{2}(2 k+1) \theta \\
& \Longrightarrow \sin ^{2}(2 k+1) \theta \geq 1-\frac{M}{N}
\end{aligned}
$$

Step e): Apply the QFT which performs the following action

$$
\left|z>\rightarrow \frac{1}{\sqrt{N}} \sum_{y=0}^{N-1} e^{-2 \pi i z y / N}\right| y>
$$

After the application of the QFT to the state $\left|\psi_{k}\right\rangle$, letting $\omega=e^{-2 \pi i / N}$, we have

$$
\left|\phi_{k}>=\frac{a_{k}}{\sqrt{N}} \sum_{z \in A} \sum_{y=0}^{N-1} \omega^{z y}\right| y>+\frac{b_{k}}{\sqrt{N}} \sum_{z \notin A} \sum_{y=0}^{N-1} \omega^{z y} \mid y>
$$

After interchanging the order of summation, we have

$$
\left|\phi_{k}>=\sum_{y=0}^{N-1}\left[\frac{a_{k}}{\sqrt{N}} \sum_{z \in A} \omega^{z y}+\frac{b_{k}}{\sqrt{N}} \sum_{z \notin A} \omega^{z y}\right]\right| y>
$$

Steps f) to i): Measure with respect to the standard basis to yield a integer $y \in\{0,1, \ldots, N-1\}$ from which we can determine the period $\mathrm{P}$ using the continued fraction method.

The amplitude $A m p(y)$ of $\mid y>$ is given by

$$
\begin{aligned}
A m p(y) & =\frac{a_{k}}{\sqrt{N}} \sum_{z \in A} \omega^{z y}+\frac{b_{k}}{\sqrt{N}} \sum_{z \notin A} \omega^{z y} \\
& =\frac{\left(a_{k}-b_{k}\right)}{\sqrt{N}} \sum_{z \in A} \omega^{z y}+\frac{b_{k}}{\sqrt{N}} \sum_{z=0}^{N-1} \omega^{z y} \\
& =\frac{\left(a_{k}-b_{k}\right)}{\sqrt{N}} \sum_{r=0}^{M-1} \omega^{(s+r P) y}+\frac{b_{k}}{\sqrt{N}} \sum_{z=0}^{N-1} \omega^{z y} \\
& =\frac{\left(a_{k}-b_{k}\right)}{\sqrt{N}} \omega^{s y} \sum_{r=0}^{M-1} \omega^{r P y}+\frac{b_{k}}{\sqrt{N}} \sum_{z=0}^{N-1} \omega^{z y}
\end{aligned}
$$

We calculate the $\operatorname{Pr}(y)$ for the following cases:

a) $y=0$

b) $P y=0 \bmod N$ and $y \neq 0$

c) $P y \neq 0 \bmod N$

9.1. Amplified-QFT Analysis: $\mathbf{y}=\mathbf{0}$. We have 
THE AMPLIFIED QUANTUM FOURIER TRANSFORM:SOLVING THE LOCAL PERIOD PROBLEM

$$
\begin{aligned}
A m p(y) & =\frac{a_{k}}{\sqrt{N}} \sum_{z \in A} \omega^{z y}+\frac{b_{k}}{\sqrt{N}} \sum_{z \notin A} \omega^{z y} \\
& =\frac{1}{\sqrt{N}}\left(M a_{k}+(N-M) b_{k}\right) \\
& =\frac{1}{\sqrt{N}}\left[\frac{M}{\sqrt{M}} \sin (2 k+1) \theta+\frac{N-M}{\sqrt{N-M}} \cos (2 k+1) \theta\right] \\
& =\sqrt{\frac{M}{N}} \sin (2 k+1) \theta+\sqrt{1-\frac{M}{N}} \cos (2 k+1) \theta \\
& =\sin \theta \sin (2 k+1) \theta+\cos \theta \cos (2 k+1) \theta \\
& =\cos (2 k \theta)
\end{aligned}
$$

We have

$$
\operatorname{Pr}(y=0)=\cos ^{2}(2 k \theta)
$$

9.2. Amplified-QFT Analysis: $P y=0 \bmod N, y \neq 0$. Using the fact that

$$
\sum_{z=0}^{N-1} \omega^{z y}=\frac{1-\omega^{N y}}{1-\omega^{y}}=0, w^{y} \neq 1
$$

we have

$$
\begin{aligned}
A m p(y) & =\frac{\left(a_{k}-b_{k}\right)}{\sqrt{N}} \omega^{s y} \sum_{r=0}^{M-1} \omega^{r P y}+\frac{b_{k}}{\sqrt{N}} \sum_{z=0}^{N-1} \omega^{z y} \\
& =\frac{\left(a_{k}-b_{k}\right)}{\sqrt{N}} \omega^{s y} \sum_{r=0}^{M-1} \omega^{r P y} \\
& =\frac{\left(a_{k}-b_{k}\right)}{\sqrt{N}} \omega^{s y} M \\
& =\frac{M w^{s y}}{\sqrt{N M}} \sin (2 k+1) \theta-\frac{M w^{s y}}{\sqrt{N(N-M)}} \cos (2 k+1) \theta \\
& =\omega^{s y} \sqrt{\frac{M}{N}}\left(\sin (2 k+1) \theta-\sqrt{\frac{M / N}{1-M / N}} \cos (2 k+1) \theta\right) \\
& =\omega^{s y} \sqrt{\frac{M}{N}}\left(\sin (2 k+1) \theta-\frac{\sin \theta}{\cos \theta} \cos (2 k+1) \theta\right) \\
& =\omega^{s y} \tan \theta \sin 2 k \theta
\end{aligned}
$$

We have

$$
\operatorname{Pr}(y)=\tan ^{2} \theta \sin ^{2} 2 k \theta
$$


DAVID J. CORNWELL

Using $k=\left\lfloor\frac{\pi}{4 \theta}\right\rfloor \Longrightarrow \frac{\pi}{4 \theta}-1 \leq k \leq \frac{\pi}{4 \theta} \Longrightarrow \frac{\pi}{2}-2 \theta \leq 2 k \theta \leq \frac{\pi}{2} \Longrightarrow \sin \left(\frac{\pi}{2}-2 \theta\right) \leq$ $\sin 2 k \theta \leq 1$ we have

$$
\begin{aligned}
\frac{\sin ^{2} \theta}{\cos ^{2} \theta} & \geq \operatorname{Pr}(y)=\tan ^{2} \theta \sin ^{2} 2 k \theta \geq \tan ^{2} \theta \sin ^{2}\left(\frac{\pi}{2}-2 \theta\right) \\
& \Longrightarrow \frac{M}{N} \frac{1}{1-\frac{M}{N}} \geq \operatorname{Pr}(y) \geq \tan ^{2} \theta \sin ^{2}\left(\frac{\pi}{2}-2 \theta\right) \\
& \Longrightarrow \frac{M}{N}\left(\frac{N}{N-M}\right) \geq \operatorname{Pr}(y) \geq \frac{\sin ^{2} \theta}{\cos ^{2} \theta} \cos ^{2} 2 \theta \\
& \Longrightarrow \frac{M}{N}\left(\frac{N}{N-M}\right) \geq \operatorname{Pr}(y) \geq \frac{\sin ^{2} \theta}{\cos ^{2} \theta}\left(2 \cos ^{2} \theta-1\right)^{2} \\
& \Longrightarrow \frac{M}{N}\left(\frac{N}{N-M}\right) \geq \operatorname{Pr}(y) \geq \frac{M}{N}\left(\frac{N}{N-M}\right)\left(1-\frac{2 M}{N}\right)^{2}
\end{aligned}
$$

9.3. Amplified-QFT Analysis: $P y \neq 0 \bmod N$. Making use of the previous results we have

$$
\begin{aligned}
A m p(y) & =\frac{\left(a_{k}-b_{k}\right)}{\sqrt{N}} \omega^{s y} \sum_{r=0}^{M-1} \omega^{r P y}+\frac{b_{k}}{\sqrt{N}} \sum_{z=0}^{N-1} \omega^{z y} \\
& =\frac{\left(a_{k}-b_{k}\right)}{\sqrt{N}} \omega^{s y} \sum_{r=0}^{M-1} \omega^{r P y} \\
& =\frac{\left(a_{k}-b_{k}\right)}{\sqrt{N}} \omega^{s y}\left[\frac{1-\omega^{M P y}}{1-\omega^{P y}}\right] \\
& =\frac{1}{M} \frac{\left(a_{k}-b_{k}\right)}{\sqrt{N}} \omega^{s y} M\left[\frac{1-\omega^{M P y}}{1-\omega^{P y}}\right] \\
& =\frac{1}{M} \omega^{s y} \tan \theta \sin 2 k \theta\left[\frac{1-\omega^{M P y}}{1-\omega^{P y}}\right]
\end{aligned}
$$

Making use of the following identity

$$
\left|1-e^{i \theta}\right|^{2}=4 \sin ^{2}(\theta / 2)
$$

we have

$$
\left|\frac{1-\omega^{M P y}}{1-\omega^{P y}}\right|^{2}=\frac{\sin ^{2}(\pi M P y / N)}{\sin ^{2}(\pi P y / N)}
$$

and so

$$
\operatorname{Pr}(y)=\frac{1}{M^{2}} \tan ^{2} \theta \sin ^{2} 2 k \theta \frac{\sin ^{2}(\pi M P y / N)}{\sin ^{2}(\pi P y / N)}
$$

Using the previous result $\frac{M}{N}\left(\frac{N}{N-M}\right) \geq \tan ^{2} \theta \sin ^{2} 2 k \theta \geq \frac{M}{N}\left(\frac{N}{N-M}\right)\left(\frac{N-2 M}{N}\right)^{2}$ and letting $R=\frac{\sin ^{2}(\pi M P y / N)}{\sin ^{2}(\pi P y / N)}$ we have

$$
\begin{aligned}
\frac{1}{M^{2}} \frac{M}{N}\left(\frac{N}{N-M}\right) R & \geq \operatorname{Pr}(y) \geq \frac{1}{M^{2}} \frac{M}{N}\left(\frac{N}{N-M}\right)\left(1-\frac{2 M}{N}\right)^{2} R \text { and so } \\
\frac{1}{N M}\left(\frac{N}{N-M}\right) R & \geq \operatorname{Pr}(y) \geq \frac{1}{N M}\left(\frac{N}{N-M}\right)\left(1-\frac{2 M}{N}\right)^{2} R
\end{aligned}
$$

We notice that if in addition $M P y=0 \bmod N$ then $\operatorname{Pr}(y)=0$. 
THE AMPLIFIED QUANTUM FOURIER TRANSFORM:SOLVING THE LOCAL PERIOD PROBLEM

9.4. Amplified-QFT Summary. The probability $\operatorname{Pr}(y)$ is given exactly by

$$
\left\{\begin{array}{ll}
\cos ^{2} 2 k \theta & \text { if } \quad y=0 \\
\tan ^{2} \theta \sin ^{2} 2 k \theta & \text { if } \quad P y=0 \bmod N, y \neq 0 \\
\frac{1}{M^{2}} \tan ^{2} \theta \sin ^{2} 2 k \theta \frac{\sin ^{2}(\pi M P y / N)}{\sin ^{2}(\pi P y / N)} & \text { if } \quad P y \neq 0 \bmod N \text { and } M P y \neq 0 \bmod N \\
0 & \text { if } \quad P y \neq 0 \bmod N \text { and } M P y=0 \bmod N
\end{array}\right\}
$$

\section{The QFT Algorithm - Detailed Analysis.}

In this section we examine the QFT algorithm in detail and produce the results for the probability of success that were summarized earlier in the paper. We just apply the QFT to the binary oracle $\mathrm{f}$, which is 1 on A and 0 elsewhere.

We begin with the following state

$$
\left|\xi>=\frac{1}{\sqrt{N}} \sum_{z=0}^{N-1}\right| z>\otimes \frac{1}{\sqrt{2}}(|0>-| 1>)
$$

and apply the unitary transform for $\mathrm{f}, U_{f}$, to this state which performs the following action:

$$
U_{f}|z>| c>=|z>| c \oplus f(z)>
$$

to get the state $|\psi\rangle$

$$
\begin{aligned}
\mid \psi & >=U_{f} \frac{1}{\sqrt{N}} \sum_{z=0}^{N-1} \mid z>\frac{1}{\sqrt{2}}(|0>-| 1>) \\
& =\frac{1}{\sqrt{N}}\left[(-1) \sum_{z \in A}\left|z>+\sum_{z \notin A}\right| z>\right] \frac{1}{\sqrt{2}}(|0>-| 1>) \\
& =\frac{1}{\sqrt{N}}\left[(-2) \sum_{z \in A}\left|z>+\sum_{z=0}^{N-1}\right| z>\right] \frac{1}{\sqrt{2}}(|0>-| 1>)
\end{aligned}
$$

Next we apply the QFT to try to find the period P, dropping $\frac{1}{\sqrt{2}}(|0>-| 1>)$.

The QFT applies the following action:

$$
\left|z>\rightarrow \frac{1}{\sqrt{N}} \sum_{y=0}^{N-1} \omega^{z y}\right| y>
$$

to get

$$
\left|\phi>=\sum_{y=0}^{N-1}\left[\frac{(-2)}{N} \sum_{z \in A} \omega^{z y}+\frac{1}{N} \sum_{z=0}^{N-1} \omega^{z y}\right]\right| y>
$$

We calculate the $\operatorname{Pr}(y)$ for the following cases:

a) $y=0$

b) $P y=0 \bmod N$ and $y \neq 0$ 
c) $P y \neq 0 \bmod N$

10.1. QFT Analysis: $y=0$. We have

$$
\begin{aligned}
A m p(y) & =\frac{(-2)}{N} \sum_{z \in A} \omega^{z y}+\frac{1}{N} \sum_{z=0}^{N-1} \omega^{z y} \\
& =\frac{(-2) M}{N}+\frac{N}{N} \\
& =1-\frac{2 M}{N}
\end{aligned}
$$

Therefore, in the QFT case, we have $\operatorname{Pr}(y=0)$ is very close to 1 and is given by

$$
\operatorname{Pr}(y=0)=1-\frac{4 M}{N}+4 \frac{M^{2}}{N^{2}}=\left(1-\frac{2 M}{N}\right)^{2}
$$

whereas in the Amplified-QFT case we have $\operatorname{Pr}(y=0)$ is given by

$$
\operatorname{Pr}(y=0)=\cos ^{2} 2 k \theta
$$

10.2. QFT Analysis: $P y=0 \bmod N, y \neq 0$. Using the fact that

$$
\sum_{z=0}^{N-1} \omega^{z y}=\frac{1-\omega^{N y}}{1-\omega^{y}}=0
$$

we have

$$
\begin{aligned}
A m p(y) & =\frac{-2}{N} \sum_{z \in A} \omega^{z y}+\frac{1}{N} \sum_{z=0}^{N-1} \omega^{z y} \\
& =\frac{-2}{N} \omega^{s y} \sum_{r=0}^{M-1} \omega^{r P y} \\
& =\frac{-2 M}{N} \omega^{s y}
\end{aligned}
$$

Therefore in the QFT case we have $\operatorname{Pr}(y)$ is given by

$$
\operatorname{Pr}(y)=4 \frac{M^{2}}{N^{2}}
$$

whereas in the Amplified-QFT case we have $\operatorname{Pr}(y)$ is given by

$$
\operatorname{Pr}(y)=\tan ^{2} \theta \sin ^{2} 2 k \theta
$$

We can determine how the increase in amplitude varies with the number of iterations $k$ of the Grover step in the Amplified-QFT by examining the ratio of the amplitudes 
THE AMPLIFIED QUANTUM FOURIER TRANSFORM:SOLVING THE LOCAL PERIOD PROBLEN及

of the Amplified-QFT case and QFT case. This ratio is given exactly by

$$
\begin{aligned}
\operatorname{AmpRatio}(y) & =\frac{\frac{\left(a_{k}-b_{k}\right)}{\sqrt{N}} \omega^{s y} M}{\frac{-2 M}{N} \omega^{s y}} \\
& =\frac{\left(a_{k}-b_{k}\right)}{-2} \sqrt{N} \\
& =\frac{1}{-2}\left[\sqrt{\frac{N}{M}} \sin (2 k+1) \theta-\sqrt{\frac{N}{N-M}} \cos (2 k+1) \theta\right] \\
& =\frac{N}{-2 M} \tan \theta \sin 2 k \theta
\end{aligned}
$$

Using $k=\left\lfloor\frac{\pi}{4 \theta}\right\rfloor$ and making use of $\frac{M}{N}\left(\frac{N}{N-M}\right) \geq \tan ^{2} \theta \sin ^{2} 2 k \theta \geq \frac{M}{N}\left(\frac{N}{N-M}\right)\left(\frac{N-2 M}{N}\right)^{2}$, we have the following inequality for the $\operatorname{Pr} \operatorname{Ratio}(y)$, the increase in the probability due to amplification:

$$
\begin{aligned}
\frac{N}{4 M}\left(\frac{N}{N-M}\right) & \geq \operatorname{Pr} \operatorname{Ratio}(y) \geq \frac{N}{4 M}\left(\frac{N}{N-M}\right)\left(1-\frac{2 M}{N}\right)^{2} \\
& \Longrightarrow \operatorname{Pr} \operatorname{Ratio}(y) \approx \frac{N}{4 M}
\end{aligned}
$$

10.3. QFT Analysis: $P y \neq 0 \bmod N$. We have

$$
\begin{aligned}
A m p(y) & =\frac{-2}{N} \sum_{z \in A} \omega^{z y}+\frac{1}{N} \sum_{z=0}^{N-1} \omega^{z y} \\
& =\frac{-2}{N} w^{s y} \sum_{r=0}^{M-1} \omega^{r P y} \\
& =\frac{-2}{N} w^{s y}\left[\frac{1-\omega^{M P y}}{1-\omega^{P y}}\right] \\
& =\frac{-2}{N} w^{s y}\left[\frac{1-\omega^{M P y}}{1-\omega^{P y}}\right]
\end{aligned}
$$

Once again, making use of the following identity

$$
\left|1-e^{i \theta}\right|^{2}=4 \sin ^{2}(\theta / 2)
$$

in the QFT case, we have $\operatorname{Pr}(y)$ is given by

$$
\operatorname{Pr}(y)=\frac{4}{N^{2}}\left[\frac{\sin ^{2}(\pi M P y / N)}{\sin ^{2}(\pi P y / N)}\right]
$$

whereas in the Amplified-QFT case we have $\operatorname{Pr}(y)$ is given by

$$
\operatorname{Pr}(y)=\frac{1}{M^{2}} \tan ^{2} \theta \sin ^{2} 2 k \theta \frac{\sin ^{2}(\pi M P y / N)}{\sin ^{2}(\pi P y / N)}
$$

We notice that if in addition $M P y=0 \bmod N$ then $\operatorname{Pr}(y)=0$. 
The ratio of the amplitudes of the Amplified-QFT case and QFT case is given exactly by

$$
\begin{aligned}
\operatorname{AmpRatio}(y) & =\frac{\frac{\left(a_{k}-b_{k}\right)}{\sqrt{N}} \omega^{s y}\left[\frac{1-\omega^{M P y}}{1-\omega^{P y}}\right]}{\frac{-2}{N} w^{s y}\left[\frac{1-\omega^{M P y}}{1-\omega^{P y}}\right]} \\
& =\frac{\left(a_{k}-b_{k}\right)}{-2} \sqrt{N} \\
& =\frac{1}{-2}\left[\sqrt{\frac{N}{M}} \sin (2 k+1) \theta-\sqrt{\frac{N}{N-M}} \cos (2 k+1) \theta\right] \\
& =\frac{N}{-2 M} \tan \theta \sin 2 k \theta
\end{aligned}
$$

We note that this ratio is the same as in that given in the previous section and is independent of $y$. The variables in this ratio do not depend in anyway on the QFT.

As in the previous section, we have the following inequality for the $\operatorname{Pr} \operatorname{Ratio}(y)$, the increase in the probability due to amplification when $k=\left\lfloor\frac{\pi}{4 \theta}\right\rfloor$ and making use of $\frac{M}{N}\left(\frac{N}{N-M}\right) \geq \tan ^{2} \theta \sin ^{2} 2 k \theta \geq \frac{M}{N}\left(\frac{N}{N-M}\right)\left(\frac{N-2 M}{N}\right)^{2}$

$$
\begin{aligned}
\frac{N}{4 M}\left(\frac{N}{N-M}\right) & \geq \operatorname{Pr} \operatorname{Ratio}(y) \geq \frac{N}{4 M}\left(\frac{N}{N-M}\right)\left(1-\frac{2 M}{N}\right)^{2} \\
& \Longrightarrow \operatorname{Pr} \operatorname{Ratio}(y) \approx \frac{N}{4 M}
\end{aligned}
$$

10.4. QFT Summary. The probability $\operatorname{Pr}(y)$ is given exactly by

$$
\left\{\begin{array}{ll}
\left(1-\frac{2 M}{N}\right)^{2} & \text { if } \quad y=0 \\
4 \frac{M^{2}}{N^{2}} & \text { if } \quad P y=0 \bmod N, y \neq 0 \\
\frac{4}{N^{2}} \frac{\sin ^{2}(\pi M P y / N)}{\sin ^{2}(\pi P y / N)} & \text { if } \quad P y \neq 0 \bmod N \text { and } M P y \neq 0 \bmod N \\
0 & \text { if } \quad P y \neq 0 \bmod N \text { and } M P y=0 \bmod N
\end{array}\right\}
$$

\section{The QHS Algorithm - Detailed Analysis}

In this section we examine the QHS algorithm in detail and produce the results for the probability of success that were summarized earlier in the paper. The QHS algorithm is a two register algorithm as follows (see ref[13] for details). We begin with $|0>| 0>$ where the first register is $n$ qubits and the second register is 1 qubit and apply the Hadamard transform to the first register to get a uniform superposition state, followed by the unitary transformation for the Oracle $f$ to get:

$$
\left|\psi>=\frac{1}{\sqrt{N}} \sum_{x=0}^{N-1}\right| x>\mid f(x)>
$$

Next we apply the QFT to the first register to get 
THE AMPLIFIED QUANTUM FOURIER TRANSFORM:SOLVING THE LOCAL PERIOD PROBLEM

$$
\begin{aligned}
\mid \psi & >=\frac{1}{\sqrt{N}} \sum_{x=0}^{N-1} \frac{1}{\sqrt{N}} \sum_{y=0}^{N-1} \omega^{x y}|y>| f(x)> \\
& =\sum_{y=0}^{N-1} \frac{1}{N} \sum_{x=0}^{N-1} \omega^{x y}|y>| f(x)> \\
& =\sum_{y=0}^{N-1} \frac{1}{N}\left|y>\sum_{x=0}^{N-1} \omega^{x y}\right| f(x)> \\
& =\sum_{y=0}^{N-1} \frac{|| \Gamma(y)>\|}{N} \mid y>\frac{\mid \Gamma(y)>}{\|\mid \Gamma(y)>\|}
\end{aligned}
$$

where

$$
\begin{aligned}
\mid \Gamma(y) & >=\sum_{x=0}^{N-1} \omega^{x y} \mid f(x)> \\
& =\sum_{x \in A} \omega^{x y}\left|1>+\sum_{x \notin A} \omega^{x y}\right| 0>
\end{aligned}
$$

and where

$$
\left\|\left.\left|\Gamma(y)>\|^{2}=\right| \sum_{x \in A} \omega^{x y}\right|^{2}+\left|\sum_{x \notin A} \omega^{x y}\right|^{2}\right.
$$

Next we make a measurement to get $y$ and find that the probability of this measurement is

$$
\begin{aligned}
\operatorname{Pr}(y) & =\frac{\|\Gamma(y)>\|^{2}}{N^{2}} \\
& =\frac{1}{N^{2}}\left|\sum_{x \in A} \omega^{x y}\right|^{2}+\frac{1}{N^{2}}\left|\sum_{x \notin A} \omega^{x y}\right|^{2}
\end{aligned}
$$

The state that we end up in is of the form

$$
|\phi>=| y>\frac{\mid \Gamma(y)>}{\|\Gamma(y)>\|}
$$

We calculate the $\operatorname{Pr}(y)$ for the following cases:
a) $y=0$
b) $P y=0 \bmod N$ and $y \neq 0$
c) $P y \neq 0 \bmod N$

11.1. QHS Analysis: $y=0$. We have

$$
\begin{aligned}
\operatorname{Pr}(y) & =\frac{1}{N^{2}}\left|\sum_{x \in A} \omega^{x y}\right|^{2}+\frac{1}{N^{2}}\left|\sum_{x \notin A} \omega^{x y}\right|^{2} \\
& =\frac{M^{2}}{N^{2}}+\frac{(N-M)^{2}}{N^{2}}=\frac{M^{2}+N^{2}-2 N M+M^{2}}{N^{2}} \\
& =1-\frac{2 M(N-M)}{N^{2}}
\end{aligned}
$$


whereas in the Amplified-QFT case we have $\operatorname{Pr}(y=0)$ is given by

$$
\operatorname{Pr}(y=0)=\cos ^{2} 2 k \theta
$$

11.2. QHS Analysis: $P y=0 \bmod N, y \neq 0$. We have

$$
\begin{aligned}
\operatorname{Pr}(y) & =\frac{1}{N^{2}}\left|\sum_{x \in A} \omega^{x y}\right|^{2}+\frac{1}{N^{2}}\left|\sum_{x \notin A} \omega^{x y}\right|^{2} \\
& =\frac{1}{N^{2}}\left|\omega^{s y} \sum_{r=0}^{M-1} \omega^{r P y}\right|^{2}+\frac{1}{N^{2}}\left|\sum_{x \notin A} \omega^{x y}\right|^{2} \\
& =\frac{1}{N^{2}}\left|\omega^{s y} \sum_{r=0}^{M-1} \omega^{r P y}\right|^{2}+\frac{1}{N^{2}}\left|-\omega^{s y} \sum_{r=0}^{M-1} \omega^{r P y}+\frac{1}{N} \sum_{x=0}^{N-1} \omega^{x y}\right|^{2} \\
& =\frac{2 M^{2}}{N^{2}}
\end{aligned}
$$

where we have used the fact that

$$
\sum_{x=0}^{N-1} \omega^{x y}=0
$$

In the Amplified-QFT case we have $\operatorname{Pr}(y)$ is given by

$$
\operatorname{Pr}(y)=\tan ^{2} \theta \sin ^{2} 2 k \theta
$$

By comparing the results of the QHS and the Amplified-QFT algorithms we have the following inequality for the $\operatorname{Pr}$ Ratio $(y)=\operatorname{Pr}(y)_{\text {Amplified-QFT }} / \operatorname{Pr}(y)_{Q H S}$, the increase in the probability due to amplification when $k=\left\lfloor\frac{\pi}{4 \theta}\right\rfloor$ and making use of $\frac{M}{N}\left(\frac{N}{N-M}\right) \geq \tan ^{2} \theta \sin ^{2} 2 k \theta \geq \frac{M}{N}\left(\frac{N}{N-M}\right)\left(\frac{N-2 M}{N}\right)^{2}$

$$
\begin{aligned}
\frac{N}{2 M}\left(\frac{N}{N-M}\right) & \geq \operatorname{Pr} \operatorname{Ratio}(y) \geq \frac{N}{2 M}\left(\frac{N}{N-M}\right)\left(1-\frac{2 M}{N}\right)^{2} \\
& \Longrightarrow \operatorname{Pr} \operatorname{Ratio}(y) \approx \frac{N}{2 M}
\end{aligned}
$$

11.3. QHS Analysis: $P y \neq 0 \bmod N$. We have

$$
\begin{aligned}
\operatorname{Pr}(y) & =\frac{1}{N^{2}}\left|\sum_{x \in A} \omega^{x y}\right|^{2}+\frac{1}{N^{2}}\left|\sum_{x \notin A} \omega^{x y}\right|^{2} \\
& =\frac{1}{N^{2}}\left|\omega^{s y} \sum_{r=0}^{M-1} \omega^{r P y}\right|^{2}+\frac{1}{N^{2}}\left|\sum_{x \notin A} \omega^{x y}\right|^{2} \\
& =\frac{1}{N^{2}}\left|\omega^{s y} \sum_{r=0}^{M-1} \omega^{r P y}\right|^{2}+\frac{1}{N^{2}}\left|-\omega^{s y} \sum_{r=0}^{M-1} \omega^{r P y}+\frac{1}{N} \sum_{x=0}^{N-1} \omega^{x y}\right|^{2} \\
& =\frac{1}{N^{2}}\left|\omega^{s y}\left[\frac{1-\omega^{M P y}}{1-\omega^{P y}}\right]\right|^{2}+\frac{1}{N^{2}}\left|-\omega^{s y}\left[\frac{1-\omega^{M P y}}{1-\omega^{P y}}\right]\right|^{2} \\
& =\frac{2}{N^{2}} \frac{\sin ^{2}(\pi M P y / N)}{\sin ^{2}(\pi P y / N)}
\end{aligned}
$$


THE AMPLIFIED QUANTUM FOURIER TRANSFORM:SOLVING THE LOCAL PERIOD PROBLEAף

where we have used the fact that

$$
\sum_{x=0}^{N-1} \omega^{x y}=0
$$

and that

$$
\left|1-e^{i \theta}\right|^{2}=4 \sin ^{2}(\theta / 2)
$$

In the Amplified-QFT case we have $\operatorname{Pr}(y)$ is given by

$$
\operatorname{Pr}(y)=\frac{1}{M^{2}} \tan ^{2} \theta \sin ^{2} 2 k \theta \frac{\sin ^{2}(\pi M P y / N)}{\sin ^{2}(\pi P y / N)}
$$

We notice that if in addition $M P y=0 \bmod N$ then $\operatorname{Pr}(y)=0$.

By comparing the results of the QHS and the Amplified-QFT algorithms we have the following inequality for the $\operatorname{Pr}$ Ratio $(y)=\operatorname{Pr}(y)_{\text {Amplified-QFT }} / \operatorname{Pr}(y)_{Q H S}$, the increase in the probability due to amplification when $k=\left\lfloor\frac{\pi}{4 \theta}\right\rfloor$ and making use of $\frac{M}{N}\left(\frac{N}{N-M}\right) \geq \tan ^{2} \theta \sin ^{2} 2 k \theta \geq \frac{M}{N}\left(\frac{N}{N-M}\right)\left(\frac{N-2 M}{N}\right)^{2}$

$$
\begin{aligned}
\frac{N}{2 M}\left(\frac{N}{N-M}\right) & \geq \operatorname{Pr} \operatorname{Ratio}(y) \geq \frac{N}{2 M}\left(\frac{N}{N-M}\right)\left(1-\frac{2 M}{N}\right)^{2} \\
& \Longrightarrow \operatorname{Pr} \operatorname{Ratio}(y) \approx \frac{N}{2 M}
\end{aligned}
$$

11.4. QHS Summary. The $\operatorname{Pr}(y)$ in the QHS case is:

$$
\left\{\begin{array}{ll}
1-\frac{2 M(N-M)}{N^{2}} & \text { if } \quad y=0 \\
\frac{2 M^{2}}{N^{2}} & \text { if } \quad P y=0 \bmod N, y \neq 0 \\
\frac{2}{N^{2}} \frac{\sin ^{2}(\pi M P y / N)}{\sin ^{2}(\pi P y / N)} & \text { if } \quad P y \neq 0 \bmod N \text { and } M P y \neq 0 \bmod N \\
0 & \text { if } \quad P y \neq 0 \bmod N \text { and } M P y=0 \bmod N
\end{array}\right\}
$$

12. Recovering the Period $\mathbf{P}$ and the Offset $\mathbf{s}$

As in Shor's algorithm, we use the continued fraction expansion of $y / N$ to find the period $P$, where $y$ is a measured value such that $y / N$ is close to $d / P$ and $(d, P)=1$ . See ref[2] and ref[3]for details which we provide below.

Let $\{a\}_{N}$ be the residue of $a \bmod N$ of smallest magnitude such that $-N / 2<$ $\{a\}_{N}<N / 2$. Let $S_{N}=\{0,1, \ldots, N-1\}, S_{P}=\left\{d \in S_{N}: 0 \leq d<P\right\}$ and $Y=\{y \in$ $\left.S_{N}:|P y| \leq P / 2\right\}$. Then the map $Y \rightarrow S_{P}$ given by $y \rightarrow d=d(y)=\operatorname{round}(P y / N)$ with inverse $y=y(d)=\operatorname{round}(N d / P)$ is a bijection and $\{P y\}_{N}=P y-N d(y)$. In addition the following two sets are in 1-1 correspondence $\{y / N: y \in Y\}$ and $\{d / P: 0 \leq d<P\}$.

We make use of the following theorem from the theory of continued fractions ref[5] (Theorem 184 p.153):

Theorem 1. Let $x$ be a real number and let $a$ and $b$ be integers with $b>0$. If $\left|x-\frac{a}{b}\right| \leq \frac{1}{2 b^{2}}$ then the rational $a / b$ is a convergent of the continued fraction expansion of $x$. 
Corollary 1. If $P^{2} \leq N$ and $\left|\{P y\}_{N}\right| \leq \frac{P}{2}$ then $d(y) / P$ is a convergent of the continued fraction expansion of $y / N$.

Proof. Since $\{P y\}_{N}=P y-N d(y)$ we have

$|P y-N d(y)| \leq \frac{P}{2}$ or

$\left|\frac{y}{N}-\frac{d(y)}{P}\right| \leq \frac{1}{2 N} \leq \frac{1}{2 P^{2}}$

and we can apply Theorem 1 so that $d / P$ is a convergent of the continued fraction expansion of $y / N$.

Since we know $y$ and $N$ we can find the continued fraction expansion of $y / N$. However we also need that $(d, P)=1$ in order that $d / P$ is a convergent and enabling us to read off $P$ directly. The probability that $(d, P)=1$ is $\varphi(P) / P$ where $\varphi(P)$ is Euler's totient function. If $P$ is prime we get $(d, P)=1$ trivially.

By making use of the following Theorem it can be shown that $\frac{\varphi(P)}{P} \geq \frac{e^{-\gamma}-\epsilon(P)}{\ln 2} \frac{1}{\ln \ln N}$ , where $\epsilon(P)$ is a monotone decreasing sequence converging to zero.

Theorem 2. $\liminf \frac{\varphi(N)}{N / \ln \ln N}=e^{-\gamma}$

where $\gamma=0.57721566$ is Euler's constant and where $e^{-\gamma}=0.5614594836$.

This may cause us to repeat the experiment $\Omega\left(\frac{1}{\ln \ln N}\right)$ times in order to get $(d, P)=1$.

We note that we needed to add a condition on the period $P$ that $P^{2} \leq N$ or $P \leq \sqrt{N}$ in order for the proof of the corollary to work.

12.1. Testing if $P_{1}=P$ when $s$ is known or is 0 . We can easily test if $s=0$ by checking to see if $f(0)=1$.

Now given a putative value of the period $P_{1}$ and a known offset or shift $s$, how can we test whether $P_{1}=P$ ?

Assuming we have access to the Oracle to test individual values, we can confirm $f(s)=1$ since $s$ is known. We will show that if $f\left(s+P_{1}\right)=1$ and $f\left(s+(M-1) P_{1}\right)=$ 1 then $P_{1}=P$.

Case 1: If $P_{1}>P$ then $s+(M-1) P_{1}>s+(M-1) P$. But $s+(M-1) P$ is the largest index $x$ such that $f(x)=1$. Therefore if $P_{1}>P$ we must have $f\left(s+(M-1) P_{1}\right)=0$.

Case 2: If $0<P_{1}<P$ then $s<s+P_{1}<s+P$ but between $s$ and $P$ there are no other values $x$ such that $f(x)=1$. Therefore if $0<P_{1}<P$ we must have $f\left(s+P_{1}\right)=0$.

Therefore if $f(s)=1, f\left(s+P_{1}\right)=1$ and $f\left(s+(M-1) P_{1}\right)=1$ we must have $P_{1}=P$.

12.2. Testing if $\left(s_{1}, P_{1}\right)=(s, P)$ when $s$ is from a small known set and $s \neq 0$. If we assume $s$ is unknown and $s \neq 0$ but is from a small known set of possible values such that we can exhaust over this set on a classical computer and we are given a putative value of the period $P_{1}$, how can we test whether a pair of values $\left(s_{1}, P_{1}\right)$ is the correct pair $(s, P)$ ?

We need only test whether $f\left(s_{1}\right)=1, f\left(s_{1}+P_{1}\right)=1$ and $f\left(s_{1}+(M-1) P_{1}\right)=1$ where $\mathrm{M}$ is assumed known.

Case 1: If $s_{1}<s$ then $f\left(s_{1}\right)=0$ since $s$ is the smallest index $x$ with $f(x)=1$.

Case 2: If $s_{1}>s$ and $f\left(s_{1}\right)=1$ then $s_{1}=s+r P$ with $r>0$. If $f\left(s_{1}+P_{1}\right)=1$ then $s_{1}+P_{1}=s+t P=s_{1}+(t-r) P$ with $t>r>0$. Hence $P_{1}=(t-r) P>0$. If 
THE AMPLIFIED QUANTUM FOURIER TRANSFORM:SOLVING THE LOCAL PERIOD PROBLE2M

$f\left(s_{1}+(M-1) P_{1}\right)=1$ then $s_{1}+(M-1) P_{1}=s+r P+(M-1)(t-r) P>s+(M-1) P$ which is the largest index $x$ with $f(x)=1$. Therefore $f\left(s_{1}+(M-1) P_{1}\right)=0$.

Hence if $f\left(s_{1}\right)=1, f\left(s_{1}+P_{1}\right)=1$ and $f\left(s_{1}+(M-1) P_{1}\right)=1$ we must have $s_{1}=s$ and then by following the case when $s$ is known we must also have $P_{1}=P$.

Therefore if one or more of the values $f\left(s_{1}\right), f\left(s_{1}+P_{1}\right), f\left(s_{1}+(M-1) P_{1}\right)$ is zero, either $s_{1}$ or $P_{1}$ is wrong. For a given $P_{1}$ we must exhaust over all possible values of $s$ before we can be sure that $P_{1} \neq P$. For in the case that $P_{1} \neq P$, we will have for every possible $s_{1}$ that at least one of the values $f\left(s_{1}\right), f\left(s_{1}+P_{1}\right)$, $f\left(s_{1}+(M-1) P_{1}\right)$ is zero. In such a case we must try another putative $P_{1}$.

12.3. Finding $s \neq 0$ using a Quantum Computer. We can assume $s \neq 0$ as the case $s=0$ is trivial and was considered above. Let $s=\alpha+\beta P$ where $\alpha=s \bmod P$ so that $0 \leq \alpha \leq P-1$ and $0 \leq \alpha+\beta P+(M-1) P \leq N-1$.

We assume we are given the correct value of $P$. If $P$ is wrong, it will be detected in the algorithm.

Step 1:

We create an initial superposition on $N$ values

$$
\left|\psi_{1}>=\frac{1}{\sqrt{N}} \sum_{x=0}^{N-1}\right| x>
$$

and apply the Oracle $f$ and put this into the amplitude. We then apply Grover without measurement to amplify the amplitudes and we have the following state

$$
\left|\psi_{1}>=a_{k} \sum_{\times \in A}\right| x>+b_{k} \sum_{x \notin A} \mid x>
$$

where

$$
a_{k}=\frac{1}{\sqrt{M}} \sin (2 k+1) \theta, b_{k}=\frac{1}{\sqrt{N-M}} \cos (2 k+1) \theta
$$

are the appropriate amplitudes of the states and where

$$
\sin \theta=\sqrt{M / N}, \cos \theta=\sqrt{1-M / N}
$$

Next we measure the register and with probability exceeding $1-M / N$ we will measure a value $x_{1} \in A$ where $x_{1}=s+r_{1} P$ with $0 \leq r_{1} \leq M-1$. Note that the total probability of the set $\mathrm{A}$ is given by

$$
\begin{aligned}
\operatorname{Pr}(x & \in A)=M\left(\frac{1}{\sqrt{M}} \sin (2 k+1) \theta\right)^{2}=\sin ^{2}(2 k+1) \theta=1-\cos ^{2}(2 k+1) \theta \\
& \Longrightarrow \operatorname{Pr}(x \in A)=\sin ^{2}(2 k+1) \theta \geq 1-\frac{M}{N}
\end{aligned}
$$

Now using our measured value $x_{1}=s+r_{1} P$ with $0 \leq r_{1} \leq M-1$ we check that $f\left(x_{1}\right)=1$ and $f\left(x_{1}-P\right)=1$. If $f\left(x_{1}-P\right)=0$ then either the value of $P$ we are using is wrong or we have $r_{1}=0$ and $x_{1}=s$. If we test $f(s)=1, f(s+P)=1$ and $f(s+(M-1) P)=1$ then we have the correct $P$ and $s$ otherwise $P$ is wrong. So assuming $f\left(x_{1}-P\right)=1$ we must have either the correct $P$ or a multiple of $P$. We can use the procedure in Step 2 or Step 2' to find $s$. The method in Step 2 uses the Exact Quantum Counting algorithm to find $s$ (See ref[11] for details). The method in Step 2' uses a method of decreasing sequence of measurements to find $s$.

Step 2 (using the Exact Quantum Counting algorithm): 
Let $T$ be such that $T \geq M$ is the smallest power of 2 greater than $M$. We form a superposition

$$
\left|\varphi_{1}>=\frac{1}{\sqrt{T}} \sum_{x=0}^{T-1}\right| x>\mid 0>
$$

and apply the function $g(x)=\operatorname{Max}\left(0, x_{1}-(x+1) P\right)$ where $x_{1}=s+r_{1} P$ is our measured value, with $0 \leq r_{1} \leq M-1$ and put the values of $g(x)$ into the second register to get

$$
\left|\varphi_{2}>=\frac{1}{\sqrt{T}} \sum_{x=0}^{T-1}\right| x>\mid g(x)>
$$

Notice that as $x$ increases from $0, g(x)$ is a decreasing sequence $s+r P$ with $r=$ $\left(r_{1}-x-1\right)$. When $g(x)$ dips below 0 we set $g(x)=0$ to ensure $g(x) \geq 0$. Now we apply $f$ to $g(x)$ and put the results into the amplitude to get

$$
\left|\varphi_{3}>=\frac{1}{\sqrt{T}} \sum_{x=0}^{T-1}(-1)^{f(g(x))}\right| x>\mid g(x)>
$$

Notice that $f(g(x))=1$ when $s \leq g(x)<s+r_{1} P$ and is 0 elsewhere. We apply the exact quantum counting algorithm which determines how many values $f(g(x))=1$.Let this total be $R$. If $P$ is correct we expect $R=r_{1}$ and we can determine $s=x_{1}-R P=s+r_{1} P-R P$. We can then test if we have the correct pair of values $s, P$ by testing whether $f(s)=1, f(s+P)=1$ and $f(s+(M-1) P)=1$. If this test fails then $P$ must be an incorrect value and we must repeat the period finding algorithm.

We use Theorem 8.3.4 of ref[11]: The Exact Quantum Counting algorithm requires an expected number of applications of $U_{f}$ in $O(\sqrt{(R+1)(T-R+1)}$ and outputs the correct value $R$ with probability at least $2 / 3$.

Step 2' (decreasing sequence of measurements method):

Let $T$ be such that $T \geq M$ is the smallest power of 2 greater than $M$. We form a superposition

$$
\left|\varphi_{1}>=\frac{1}{\sqrt{T}} \sum_{x=0}^{T-1}\right| x>\mid 0>
$$

and apply the function $g(x)=\operatorname{Max}\left(0, x_{1}-(x+1) P\right)$ where $x_{1}=s+r_{1} P$ with $0 \leq r_{1} \leq M-1$ and put these values into the second register to get

$$
\left|\varphi_{2}>=\frac{1}{\sqrt{T}} \sum_{x=0}^{T-1}\right| x>\mid g(x)>
$$

Notice that as $x$ increases from $0, g(x)$ is a decreasing sequence $s+r P$ with $r=\left(r_{1}-x-1\right)$. When $g(x)$ dips below 0 we set $g(x)=0$ to ensure $g(x) \geq 0$. Now we apply $f$ to $g(x)$ and put the results into the third register and then into the amplitude.

$$
\left|\varphi_{3}>=\frac{1}{\sqrt{T}} \sum_{x=0}^{T-1}(-1)^{f(g(x))}\right| x>\mid g(x)>
$$


THE AMPLIFIED QUANTUM FOURIER TRANSFORM:SOLVING THE LOCAL PERIOD PROBLE23

Notice that $f(g(x))=1$ when $s \leq g(x)<s+r_{1} P$ and is 0 elsewhere.

We then run Grover without measurement to amplify the amplitudes and measure the second register containing $g(x)$.

With probability close to 1 we will measure a new value $x_{2}=s+r_{2} P$ with $0 \leq r_{2}<r_{1}$. We test the values $f\left(x_{2}\right)=1$ and $f\left(x_{2}-P\right)=1$. If $f\left(x_{2}-P\right)=0$ then either the value of $P$ we are using is wrong or we have $r_{2}=0$ and $x_{2}=s$. If we test $f(s)=1, f(s+P)=1$ and $f(s+(M-1) P)=1$ then we have the correct $P$ and $s$ otherwise $P$ is wrong. So assuming $f\left(x_{2}-P\right)=1$ we must have either the correct $P$ or a multiple of $P$. We repeat this algorithm and go to Step 2' replacing the value $x_{1}$ in the function $g(x)$ with $x_{2}$ etc. As we repeat the algorithm we will measure a decreasing sequence of values $x_{1}, x_{2} \ldots$ that converges to $s$. This procedure will eventually terminate with the correct pair of values $P$ and $s$ or we will determine that we have been using an incorrect value of $P$ and we must repeat the quantum algorithm for finding putative $P$ and repeat the process.

How many times do we expect to repeat Step 2'? When we make our first measurement we expect $r_{1}=M / 2$. For our second measurement we expect $r_{2}=$ $r_{1} / 2$ etc. Therefore we expect to repeat this algorithm $O\left(\ln _{2}(M)\right)$ times.

\section{Replacing the QFT With a General Unitary Transform U}

In general, if we had any Oracle $f$ which is 1 on a set of labels $A$ and 0 elsewhere and we replaced the QFT with any unitary transform $U$ which performs the following

$$
\left|z>\rightarrow \frac{1}{\sqrt{N}} \sum_{y=0}^{N-1} \alpha(y, z)\right| y>
$$

we can compute the AmpRatio(y) $=\frac{\text { Amplified-Amplitude }(U)}{\operatorname{Amplitude}(U)}$ as follows.

As before, we have the following state after applying $U_{f}$ :

$$
\mid \psi>=\frac{1}{\sqrt{N}}\left[(-2) \sum_{z \in A}\left|z>+\sum_{z=0}^{N-1}\right| z>\right]
$$

Next we apply the general unitary transform $U$ to obtain the state

$$
U\left|\psi>=\sum_{y=0}^{N-1}\left[\frac{(-2)}{N} \sum_{z \in A} \alpha(y, z)+\frac{1}{N} \sum_{z=0}^{N-1} \alpha(y, z)\right]\right| y>
$$

In the Amplified-U case we apply Grover without measurement followed by $U$ we obtain the state

$$
\left|\phi_{k}>=\sum_{y=0}^{N-1}\left[\frac{\left(a_{k}-b_{k}\right)}{\sqrt{N}} \sum_{z \in A} \alpha(y, z)+\frac{b_{k}}{\sqrt{N}} \sum_{z=0}^{N-1} \alpha(y, z)\right]\right| y>
$$

If $\sum_{z=0}^{N-1} \alpha(y, z)=0$ and $\sum_{z \in A} \alpha(y, z) \neq 0$ we get the same AmpRatio(y) formula that we obtained when $U=Q F T$ 


$$
\begin{aligned}
\operatorname{AmpRatio}(y) & =\frac{\frac{\left(a_{k}-b_{k}\right)}{\sqrt{N}} \sum_{z \in A} \alpha(y, z)+\frac{b_{k}}{\sqrt{N}} \sum_{z=0}^{N-1} \alpha(y, z)}{\frac{(-2)}{N} \sum_{z \in A} \alpha(y, z)+\frac{1}{N} \sum_{z=0}^{N-1} \alpha(y, z)} \\
& =\frac{\frac{\left(a_{k}-b_{k}\right)}{\sqrt{N}} \sum_{z \in A} \alpha(y, z)}{\frac{(-2)}{N} \sum_{z \in A} \alpha(y, z)} \\
& =\frac{\frac{\left(a_{k}-b_{k}\right)}{\sqrt{N}}}{\frac{(-2)}{N}} \\
& =\frac{\left(a_{k}-b_{k}\right)}{-2} \sqrt{N} \\
& =\frac{1}{-2}\left[\sqrt{\frac{N}{M}} \sin (2 k+1) \theta-\sqrt{\frac{N}{N-M}} \cos (2 k+1) \theta\right] \\
& =\frac{N}{-2 M} \tan \theta \sin 2 k \theta
\end{aligned}
$$

This gives

$$
\operatorname{Pr} \operatorname{Ratio}(y)=\frac{N^{2}}{4 M^{2}} \tan ^{2} \theta \sin ^{2} 2 k \theta
$$

As in the case when $\mathrm{U}=\mathrm{QFT}$, we have the following inequality for the $\operatorname{Pr} \operatorname{Ratio}(y)$ for a general $\mathrm{U}$, the increase in the probability due to amplification when $k=\left\lfloor\frac{\pi}{4 \theta}\right\rfloor$ and making use of $\frac{M}{N}\left(\frac{N}{N-M}\right) \geq \tan ^{2} \theta \sin ^{2} 2 k \theta \geq \frac{M}{N}\left(\frac{N}{N-M}\right)\left(\frac{N-2 M}{N}\right)^{2}$

$$
\begin{aligned}
\frac{N}{4 M}\left(\frac{N}{N-M}\right) & \geq \operatorname{Pr} \operatorname{Ratio}(y) \geq \frac{N}{4 M}\left(\frac{N}{N-M}\right)\left(1-\frac{2 M}{N}\right)^{2} \\
& \Longrightarrow \operatorname{Pr} \operatorname{Ratio}(y) \approx \frac{N}{4 M}
\end{aligned}
$$

\section{References}

[1] Nakahara and Ohmi, "Quantum Computing: From Linear Algebra to Physical Realizations", CRC Press (2008).

[2] S. Lomonaco, "Shor's Quantum Factoring Algorithm," AMS PSAPM, vol. 58, (2002), 161-179.

[3] P. Shor, "Polynomial time algorithms for prime factorization and discrete logarithms on a quantum computer", SIAM J. on Computing, 26(5) (1997) pp14841509 (quant-ph/9508027).

[4] L. Grover, "A fast quantum mechanical search algorithm for database search", Proceedings of the 28th Annual ACM Symposium on Theory of Computing (STOC 1996), (1996) 212-219.

[5] Hardy and Wright "An Introduction to the Theory of Numbers", Oxford Press Fifth Edition (1979).

[6] S. Lomonaco and L. Kauffman, "Quantum Hidden Subgroup Algorithms: A Mathematical Perspective," AMS CONM, vol. 305, (2002), 139-202.

[7] S. Lomonaco, "Grover's Quantum Search Algorithm," AMS PSAPM, vol. 58, (2002), 181-192. 
THE AMPLIFIED QUANTUM FOURIER TRANSFORM:SOLVING THE LOCAL PERIOD PROBLE23

[8] S. Lomonaco and L. Kauffman, "Is Grover's Algorithm a Quantum Hidden Subgroup Algorithm?," Journal of Quantum Information Processing, Vol. 6, No. 6, (2007), 461-476.

[9] G. Brassard, P. Hoyer, M. Mosca and A. Tapp, "Quantum Amplitude Amplification and Estimation", AMS CONM, vol 305, (2002), 53-74.

[10] M. Nielsen and I. Chuang, "Quantum Computation and Quantum Information", Cambridge University Press (2000).

[11] P. Kaye, R. Laflamme and M. Mosca, "An Introduction to Quantum Computing", Oxford University Press (2007).

[12] N. Yanofsky and M. Mannucci, "Quantum Computing For Computer Scientists", Cambridge University Press (2008).

[13] S. Lomonaco, "A Lecture on Shor's Quantum Factoring Algorithm Version 1.1" quant-ph/0010034v1 9 Oct 2000.

Current address: David J. Cornwell (PhD Student), Department of Mathematics, University of Maryland Baltimore County, 1000 Hilltop Circle, Baltimore, MD 21250

E-mail address: David J. Cornwell: dave.cornwell@yahoo.com 